\title{
ARTIGO ORIGINAL Funções executivas, atividades da vida diária e habilidade motora de idosos com doenças neurodegenerativas
}

\author{
Executive functions, activities of daily living and motor skill \\ of elderly people with neurodegenerative diseases
}

Lilian Assunção Felippe', Renata Terra de Oliveira', Milena Garcia², Tânia Cristina Dias da Silva-Hamu³, Suhaila Mahmoud Smaili Santos ${ }^{4}$, Gustavo Christofoletti $^{5}$

\section{RESUMO}

Objetivo: Analisar as funções executivas de idosos com doença de Parkinson (DP - com e sem quadro demencial) e doença de Alzheimer (DA), e confrontar os escores dos participantes no que se refere às atividades funcionais da vida diária e à habilidade motora em situações de dupla tarefa. Métodos: Sob um desenho transversal, 54 idosos foram divididos em quatro grupos: G1, composto por 11 sujeitos com DP; G2, formado por 10 sujeitos com demência de Parkinson; G3, composto por 13 participantes com DA; e G4, formado por 20 idosos saudáveis. Os procedimentos metodológicos envolveram análise das funções cognitivas pré-frontais dos sujeitos, da realização das atividades da vida diária e da habilidade motora em situações de dupla tarefa. A análise dos dados envolveu a estatística descritiva (média e erro-padrão) e inferencial (teste ANOVA e pós-teste de Scheffé), admitindo significância de $5 \%$ ( $p<0,05)$ e intervalo de confiança de 95\%. Resultados: As funções cognitivas pré-frontais apresentaram diferença significativa entre os grupos, sobretudo nas comparações envolvendo G2 e G3, em relação a G1 e G4 ( $p=0,001)$. Os grupos com déficit cognitivo apresentaram pior rendimento na realização das atividades da vida diária, com menor escore do $G 2$, na qual há junção de déficit cognitivo e motor ( $p=0,001$ ). Em situações de dupla tarefa,

\section{Palavras-chave}

Doença de Parkinson, doença de Alzheimer, função executiva, atividade motora.
G2 e G3 apresentaram maiores dificuldades que os demais grupos $(p<0,05)$. Conclusão: Distúrbios pré-frontais repercutem negativamente nas atividades funcionais e na habilidade psicomotora dos indivíduos. Quando não vinculado a quadro demencial, os pacientes com DP apresentaram escores cognitivos pré-frontais e independência funcional semelhantes aos de idosos saudáveis.
1 Universidade Federal de Mato Grosso do Sul (UFMS), Programa de Pós-Graduação em Saúde e Desenvolvimento da Região Centro-Oeste. 2 UFMS, Faculdade de Medicina.

3 Universidade Estadual de Goiás (UEG), Departamento de Fisioterapia.

4 Universidade Estadual de Londrina (UEL), Centro de Ciências Biológicas e da Saúde, Departamento de Fisioterapia.

5 UFMS, Centro de Ciências Biológicas e da Saúde. UFMS, Programa de Pós-graduação em Saúde e Desenvolvimento da Região Centro-0este.

Endereço para correspondência: Gustavo Christofoletti

Universidade Federal de Mato Grosso do Sul,

Centro de Ciências Biológicas e da Saúde, Curso de Fisioterapia

Av. Costa e Silva, s/n, Setor Universitário, Caixa Postal 549

79060-900 - Campo Grande, MS, Brasil

Telefone: (67) 9638-5040

E-mail: g.christofoletti@ufms.br 


\section{Keywords}

Parkinson disease, Alzheimer disease, executive function, motor activity.

\section{ABSTRACT}

Objective: To analyze the executive functions of elderly people with Parkinson's (PD - with and without dementia) and Alzheimer's disease (AD), and to compare the independence of the subjects on the activities of daily living and their performance under dual-task conditions. Methods: This cross-sectional design study assessed 54 subjects divided into four groups: G1, formed by 11 subjects with PD; G2, formed by 10 subjects with Parkinson's dementia; $G 3$ consisting of 13 participants with AD; and G4, formed by 20 healthy control peers. The methodological procedures involved the analysis of prefrontal cognitive functions, the activities of daily living and the performance of the subjects under motor and cognitive dual-task conditions. Data analysis involved descriptive (mean and standard error) and inferential statistics (ANOVA with Scheffé post hoc test), assuming a significance level of 5\% ( $p<0.05$ ) and a confidence interval of $95 \%$. Results: The prefrontal cognitive functions showed significant differences between groups, especially in comparison involving G2 and G3 with G1 and G4 ( $p=0.001)$. The groups with cognitive decline had presented poorest performance in carrying out the activities of daily living, with worst score of $G 2$, where it has the association of motor and cognitive deficits ( $p=0.001$ ). In situations of dual-task, $G 2$ and $G 3$ showed inferior performance than the other groups. Conclusion: Prefrontal cognitive disorders negatively affect the functional activities and psychomotor skills of individuals. When not associated to dementia, PD patients have showed prefrontal scores and functional independence similar to healthy elderly.

\section{INTRODUÇÃO}

O aumento da expectativa de vida da população, associado a índices crescentes de qualidade de vida, gera, como desfecho, maior longevidade da sociedade moderna. O perfil nosológico das afecções relacionadas ao envelhecimento humano envolve características crônicas, predominantemente de origem cardiovascular, e altamente incapacitantes. As afecções neurodegenerativas, comumente catalogadas na doença de Parkinson (DP) e na demência do tipo Alzheimer (DA), são menos frequentes, mas, em virtude de sua incidência crescente com a idade e da exclusão social associada, têm grande importância'.

Ainda que a DP seja caracterizada como uma afecção primariamente subcortical (substância negra mesencefálica), com manifestações inicial e predominantemente motoras, e a DA apresente comprometimento primariamente cortical (sistema entorrinal-hipocampal e neocortical), com predomínio de alterações cognitivas e psíquico-comportamentais, ambas são doenças graves que oneram o paciente e seus familiares, terminando por comprometer a realização de tarefas cotidianas dos indivíduos ${ }^{2}$.

Por isso, para a adequada funcionalidade nas atividades da vida diária, é necessária a harmonia de fatores intrínsecos e extrínsecos, como a integralidade das funções mentais e físicas dos sujeitos, em conjunto com a adaptação do corpo às variáveis ambientais confundidoras (buracos, terrenos escorregadios, inclinações etc.) $)^{3}$.
A atuação das funções cognitivas pré-frontais na eficácia das atividades diárias já é comprovada em estudos prévios, sobretudo quando envolve atividades cotidianas complexas $^{4}$. Contudo, a sua ação vislumbrada sobre a ótica da funcionalidade e das habilidades motoras de indivíduos com doenças neurodegenerativas ainda é pouco estudada, sobretudo quando a análise envolve tarefas duplas, em que a exigência cognitiva é maior ${ }^{5}$.

Desse modo, o objetivo deste estudo foi analisar as funções executivas de idosos com DP (com e sem quadro demencial) e DA, e confrontar os escores dos participantes no que se refere às atividades funcionais da vida diária e à habilidade motora em situações de dupla tarefa.

A hipótese levantada pelos pesquisadores é a de que as funções cognitivas pré-frontais estejam comprometidas na demência de Parkinson e na DA, afetando as atividades funcionais dos sujeitos. Os autores deste estudo pressupõem que, quando submetidos à dupla tarefa, os sujeitos com DP e DA desenvolvem estratégias adaptativas diferentes, decorrentes do déficit clínico de cada condição.

\section{MÉTODOS}

\section{Casuística}

Trata-se de estudo transversal formado por sujeitos de ambos os gêneros, com idade superior a 60 anos, diagnosticados com DP (grupo 1 - G1), demência na DP (grupo 2 - G2), 
DA (grupo 3 - G3) e controles saudáveis (grupo 4 - G4). Os critérios de inclusão adotados nesta pesquisa foram: idosos diagnosticados com DP (com e sem quadro demencial associado) entre os estágios 2 e 4 no estadiamento de Hoehn$-\mathrm{Yar}^{6}$ e com comprometimento moderado na subescala motora da Unified Parkinson's Disease Rating Scale (UPDRS)7; idosos diagnosticados com DA, nos estágios moderado ou avançado no Escore Clínico de Demência8; e idosos eutróficos sem qualquer comprometimento neuromuscular e psíquico. Tanto o diagnóstico quanto o estadiamento clínico dos pacientes foram realizados por neurologistas e psiquiatras experientes na área.

Quanto aos critérios de exclusão, foram excluídos os sujeitos com comprometimento cognitivo grave (comprovado por avaliação clínica prévia e pontuação inferior a cinco no Miniexame do Estado Mental - MEEM ${ }^{9}$ ), que apresentassem disfunção osteomioarticular, amaurose congênita ou adquirida, presença de quadro vertiginoso (associado ou não ao uso medicamentoso), depressão e que não possuíssem ortostatismo e bipedestação independentes.

Após a seleção dos sujeitos, foi disponibilizada uma cópia do Termo de Consentimento, tendo sido obtida assinatura dos participantes e de seus representantes legais, nos casos envolvendo declínio cognitivo. Esta pesquisa foi aprovada por comitê de ética institucional (protocolo n 53.966).

\section{Procedimentos metodológicos}

As avaliações realizadas neste estudo envolveram a aplicação de instrumentos que analisassem as funções cognitivas pré-frontais, a habilidade motora dos sujeitos em situação de dupla tarefa e as atividades funcionais da vida diária. Todos os pacientes foram avaliados no estado on da medicação.

A avaliação das funções cognitivas pré-frontais dos participantes envolveu: 1) Montreal Cognitive Assessment (MoCA ${ }^{10}$, desenvolvido como um instrumento de rastreio cognitivo que acessa diferentes domínios, como atenção e concentração, funções executivas, memória, linguagem, habilidades visoconstrutivas, conceituação, cálculo e orientação; 2) Teste de Fluência Verbal Semântica (TFVS)"11, que abrange habilidades de memória semântica, linguagem e funções executivas; e 3) Bateria de Avaliação Frontal (BAF)'12, compreendendo seis subtestes que avaliam a formação de conceitos, programação motora, controle inibitório e autonomia.

Para avaliação da habilidade motora em situações de dupla tarefa, foi utilizado o teste Timed Get Up and Go (TUG) ${ }^{13,14}$, aplicado: $1^{\circ}$ ) de forma convencional, em que o sujeito levanta-se de uma cadeira, anda 3 metros e volta à mesma cadeira; $2^{\circ}$ ) sensibilizado com dupla tarefa motora, em que o participante realiza a atividade carregando na mão dominante um copo repleto de água; e 30) dupla tarefa cognitiva, em que o sujeito realiza a tarefa associada à contagem numérica ímpar progressiva. Nesse teste foram contabilizados o número de passos e o tempo gasto para realizar as tarefas.

No que se refere à avaliação das atividades funcionais da vida diária dos participantes, utilizamos a Escala de Autopercepção do Desempenho em Atividades de Vida Diária $(E A)^{15}$, a Escala de Atividades de Vida Diária de Katz $(E K)^{16}$ e o Índice de Pfeffer $(I P)^{17}$, respondido pelo próprio participante ou pelo familiar cuidador, nos casos envolvendo declínio cognitivo. Por meio de tais instrumentos, foi possível verificar o grau de independência dos participantes na realização das atividades básicas e instrumentais da vida diária. Todas as avaliações foram realizadas no período matutino, respeitando local pertinente aos participantes. Para evitar efeitos do cansaço, foi disponibilizado o período de descanso que o participante julgasse necessário para realizar as atividades.

\section{Análise estatística}

A análise estatística envolveu procedimentos descritivos (média, erro-padrão e análise percentual) e inferenciais. A comparação entre grupos deu-se pelo teste de análise de variâncias seguido pelo pós-teste de Scheffé, tendo sido contemplados os critérios paramétricos de normalidade e homogeneidade de variâncias. Para todas as análises, foi considerado nível de significância de 5\%, sob um intervalo de confiança de $95 \%$.

\section{RESULTADOS}

Este estudou abordou um total 109 potenciais participantes. Por causa de incapacidade motora e declínio cognitivo grave, $50,46 \%$ dos sujeitos não foram incluídos $(n=55)$, perfazendo uma amostra final de 54 sujeitos.

A amostra final foi composta por idosos de ambos os gêneros (31 homens e 23 mulheres), com amplitude de idade entre 62 e 92 anos e escolaridade variando desde não letramento até ensino superior completo. As características sociodemográficas dos participantes encontram-se descritas na tabela 1.

As análises transversais evidenciaram semelhança entre os grupos quanto a tamanho amostral $\left(X^{2}=4,51 ; p=0,218\right)$, distribuição de gênero $\left(X^{2}=1,13 ; p=0,770\right.$ para a proporção de homens; e $X^{2}=5,00 ; p=0,172$ para a proporção de muIheres), escolaridade ( $X^{2}=0,60 ; p=0,896$ para a proporção de iletrados; $X^{2}=1,43 ; p=0,699$ para a proporção de participantes com ensino fundamental; $X^{2}=3,85 ; p=0,277$ para a promoção de sujeitos com ensino médio; e $X^{2}=2,00 ; p=0,572$ para a proporção de pessoas com ensino superior completo) e idade $(F=0,10 ; p=0,957)$. Foi constatada diferença entre os grupos apenas para a proporção de pessoas com ensino primário entre os grupos $\left(X^{2}=10,8 ; p=0,012\right)$, havendo maior concentração de tais sujeitos no G3 e G4. 
Tabela 1. Características sociodemográficas da amostra

\begin{tabular}{|c|c|c|c|c|c|}
\hline Variáveis & G1 & G2 & G3 & G4 & $p$ \\
\hline Tamanho amostral & 11 & 10 & 13 & 20 & 0,218 \\
\hline \multicolumn{6}{|l|}{ Gênero } \\
\hline Masculino & 8 & 6 & 7 & 10 & 0,770 \\
\hline Feminino & 3 & 4 & 6 & 10 & 0,172 \\
\hline \multicolumn{6}{|l|}{ Escolaridade } \\
\hline Analfabetismo & 0 & 2 & 1 & 0 & 0,896 \\
\hline Ensino primário & 2 & 4 & 10 & 13 & 0,012 \\
\hline Ensino fundamental & 5 & 4 & 2 & 3 & 0,699 \\
\hline Ensino médio & 1 & 0 & 0 & 4 & 0,277 \\
\hline Ensino superior & 3 & 0 & 0 & 0 & 0,572 \\
\hline Idade (anos) & $74,27 \pm 2,58$ & $75,70 \pm 2,58$ & $75,38 \pm 2,19$ & $74,10 \pm 1,56$ & 0,957 \\
\hline MEEM (pontos) & $25,00 \pm 0,82$ & $17,60 \pm 1,52$ & $16,84 \pm 1,58$ & $23,42 \pm 1,11$ & 0,001 \\
\hline
\end{tabular}

G1: grupo doença de Parkinson; G2: grupo demência de Parkinson; G3: grupo demência de Alzheimer; G4: grupo controle; MEEM: Miniexame do Estado Mental.

Quanto ao escore do MEEM, a ANOVA indicou diferença significativa entre os grupos $(F=8,56 ; p=0,001)$, evidenciando, pelo pós-teste de Scheffé, diferença nas comparações entre $G 1$ e $G 2$ ( $p=0,005 ; 95 \% I C=1,81$ e 12,99), G1 e $G 3$ ( $p$ $=0,001 ; 95 \% \mathrm{IC}=2,91$ e 13,39), G2 e G4 ( $p=0,028 ; 95 \% \mathrm{IC}=$ $-10,35$ e -0,44), e entre $G 3$ e $G 4$ ( $p=0,04 ; 95 \% \mathrm{IC}=-10,71$ e $-1,59)$. A análise aos pares comprovou que não houve diferença significativa quanto ao escore do MEEM entre os grupos G1 e G4 ( $p=0,687 ; 95 \%$ IC $=-2,80$ e 6,80) e entre $G 2$ e $G 3$ $(p=0,982 ; 95 \%$ IC $=-4,63$ e 6,13$)$.

\section{Análise das funções cognitivas pré-frontais}

A figura 1 revela os escores obtidos pelos sujeitos nos instrumentos MoCA, BAF e TFVS. A análise vislumbrou diferença significante entre os grupos para $\operatorname{MoCA}(F=13,14 ; p=0,001)$, $F A B(F=10,5 ; p=0,001)$ e TFVS $(F=7,75 ; p=0,001)$.

Para o MoCA, houve diferenças na comparação aos pares envolvendo $\mathrm{G} 1$ e $\mathrm{G} 2$ ( $p=0,001 ; 95 \% \mathrm{IC}=5,92$ e 18,24), $\mathrm{G} 1$ e $G 3$ ( $p=0,001 ; 95 \% I C=4,48$ e 16,03), G2 e $G 4$ ( $p=0,001$; $95 \% \mathrm{IC}=-13,85$ e -2,94), e entre $\mathrm{G} 3$ e $\mathrm{G} 4(\mathrm{p}=0,009 ; 95 \% \mathrm{IC}=$ $-11,59$ e -1,55). Não houve diferença significativa nos valores do MoCA para os grupos G1 e G4 ( $p=0,263 ; 95 \%$ IC $=-1,61$ e 8,97) e $G 2$ e $G 3(p=0,846 ; 95 \%$ IC $=-7,75$ e 4,10). Em relação à BAF, a diferença envolveu as seguintes comparações: $\mathrm{G} 1 \mathrm{e}$ $G 2(p=0,001 ; 95 \% I C=3,34$ e 12,28), G1 e G3 ( $p=0,002 ; 95 \%$ $I C=1,87$ e 10,25$), G 2$ e $G 4(p=0,001 ; 95 \% I C=-10,06$ e $-2,14)$ e G3 e G4 ( $p=0,013 ; 95 \%$ IC = -7,99 e -0,71). Não houve diferença significativa de valores entre G1 e G4 ( $p=0,640 ; 95 \%$ $\mathrm{IC}=-2,13$ e 5,54$)$ e entre $\mathrm{G} 2$ e $\mathrm{G} 3(\mathrm{p}=0,704 ; 95 \% \mathrm{IC}=-6,04$ e 2,55). Quanto ao TFVS, as diferenças se estabeleceram entre G1 e G3 ( $p=0,026 ; 95 \%$ IC $=0,41$ e 8,78), G2 e G4 ( $p=0,047$; $95 \%$ IC $=-7,95$ e $-4,14)$ e $G 3$ e $G 4(p=0,001 ; 95 \%$ IC $=-9,97$ e -2,69). Não houve diferença estatisticamente significante entre $\mathrm{G} 1$ e $\mathrm{G} 2(\mathrm{p}=0,538 ; 95 \% \mathrm{IC}=-2,20$ e 6,73$), \mathrm{G} 1$ e $\mathrm{G} 4(\mathrm{p}=$ 0,$628 ; 95 \% \mathrm{IC}=-5,57$ e 2,10), e entre $\mathrm{G} 2$ e $\mathrm{G} 3$ ( $p=0,480 ; 95 \%$ $\mathrm{IC}=-1,96$ e 6,63$)$.

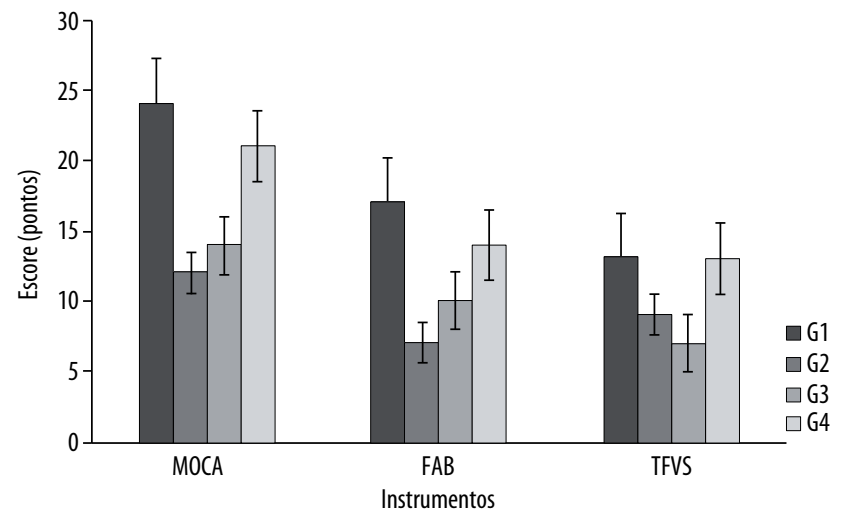

Figura 1. Valores dos grupos segundo os instrumentos de análise cognitiva.

\section{Análise das atividades da vida diária}

A tabela 2 registra os valores dos grupos, de acordo com a aplicação das escalas da vida diária. A análise transversal apontou diferença estatisticamente significativa entre os três grupos para $E A(F=5,04 ; p=0,004)$, EK $(6,63 ; p=0,001)$ e IP $(F=5,316 ; p=0,003)$.

Tabela 2. Avaliação dos grupos segundo as atividades da vida diária

\begin{tabular}{lccccc}
\hline Escalas & G1 & G2 & G3 & G4 & $\mathbf{P}$ \\
\hline EA & $120,27 \pm 10,12$ & $85,90 \pm 13,10$ & $129,23 \pm 8,23$ & $126,05 \pm 4,66$ & 0,004 \\
EK & $5,63 \pm 0,20$ & $4,40 \pm 0,61$ & $5,84 \pm 0,10$ & $5,89 \pm 0,72$ & 0,001 \\
IP & $4,09 \pm 1,88$ & $8,90 \pm 2,02$ & $12,00 \pm 2,94$ & $3,15 \pm 0,86$ & 0,003 \\
\hline EA. Escala de Autopercepcão do Desempenho em Atividades de Vida Diária: EK· Escala de Atividades de Vida Diária
\end{tabular}
列 de Katz; IP: Índice de Pfeffer; G1: grupo doença de Parkinson; G2: grupo demência de Parkinson; G3: grupo demência de Alzheimer; G4: grupo controle. 
Na análise pareada da EA, observou-se diferença estatisticamente significativa entre $\mathrm{G} 2$ e $\mathrm{G} 3(\mathrm{p}=0,007 ; 95 \% \mathrm{IC}=$ $-77,17$ e -9,48) e $\mathrm{G} 2$ e G4 ( $p=0,004 ; 95 \% \mathrm{IC}=-73,01$ e-10,68). Houve tendência de significância na comparação entre G1 e $\mathrm{G} 2$ ( $p=0,058 ; 95 \%$ IC = -0,78 e 69,53) e não houve diferença nas comparações envolvendo G1 e G3 ( $p=0,888 ; 95 \% \mathrm{IC}=$ $-41,92$ e 24,01), G1 e G4 ( $p=0,912 ; 95 \% \mathrm{IC}=-37,68$ e 22,72) e entre G3 e G4 ( $p=0,999 ; 95 \%$ IC $=-27,18$ e 30,14).

Sobre a comparação aos pares envolvendo a EK, o pósteste evidenciou diferença entre $\mathrm{G} 2$ e $\mathrm{G} 1$ ( $p=0,019 ; 95 \% \mathrm{IC}=$ $-2,30$ e -0,17), G2 e $G 3(p=0,003 ; 95 \%$ IC $=-2,47$ e $-0,41)$ e $G 2$ e $G 4(p=0,001 ; 95 \% I C=-2,44$ e -0,55). Entretanto, não houve diferença significativa nas comparações envolvendo G1 e G3 $(p=0,945 ; 95 \%$ IC $=-1,21$ e 0,79$), G 1$ e $G 4(p=0,871 ; 95 \%$ IC $=-1,18$ e 0,65$)$ e G3 e G4 ( $p=0,998 ; 95 \% \mathrm{IC}=-0,92$ e 0,81$)$.

Por fim, sobre o IP, observou-se diferença entre grupos apenas para as comparações G3 e G1 ( $p=0,036$; $95 \%$ IC $=$ 0,39 e 15,42) e G3 e G4 ( $p=0,003 ; 95 \% \mathrm{IC}=2,46$ e 15,53). As demais comparações, envolvendo os grupos G1 e G2 ( $p=$ 0,$390 ; 95 \% \mathrm{IC}=-12,82$ e 3,20), G1 e G4 ( $p=0,975 ; 95 \% \mathrm{IC}=$ $-5,79$ e 7,97$), G 2$ e $G 3(p=0,710 ; 95 \%$ IC $=-10,81$ e 4,61) e $G 2$ e G4 ( $p=0,135 ; 95 \%$ IC $=-1,20$ e 13,00), não apresentaram diferença significativa na análise em pares.

\section{Análise da habilidade motora dos sujeitos em situações de dupla tarefa}

Para verificar a funcionalidade motora nos participantes, os dados foram analisados sob um desenho transversal (com a comparação entre grupos obtidos nos escores do teste normal, com dupla tarefa motora e dupla tarefa cognitiva) e longitudinal, ao se analisarem os valores condizentes das três situações, isoladas em cada grupo. As figuras 2 e 3 contêm os escores obtidos pelos grupos segundo o tempo da tarefa e o número de passos nas três situações.

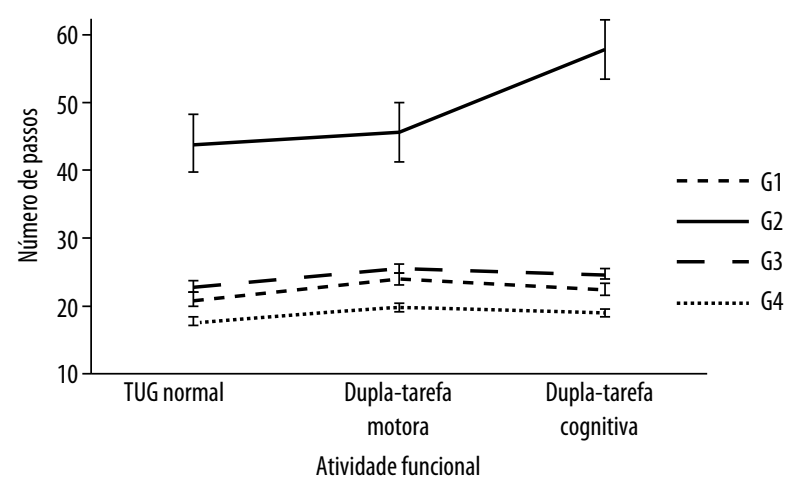

Figura 2. Número de passos realizados pelos sujeitos na atividade com e sem dupla tarefa.
Quanto à análise transversal da variável número de passos, houve diferença entre grupos na realização do teste normal $(F=7,123 ; P=0,001)$, na dupla tarefa motora $(F=9,26 ; P$ $=0,001)$ e na dupla tarefa cognitiva $(F=5,75 ; p=0,002)$. No que se refere à análise aos pares nessa variável, constatou-se diferença nas seguintes situações: $1^{\circ}$ ) no teste TUG normal: entre $G 2$ e $G 1$ ( $p=0,006 ; 95 \%$ IC = 5,43 e 41,11), entre $G 2$ e $G 3$ $(p=0,001 ; 95 \% \mathrm{IC}=3,98$ e 38,32) e entre G2 e G4 ( $p=0,001$; $95 \%$ IC $=10,93$ e 42,56$) ; 2^{\circ}$ ) na dupla tarefa motora: entre $\mathrm{G} 2$ e $G 1$ ( $p=0,002 ; 95 \% I C=6,51$ e 37,07), entre $G 2$ e $G 3$ ( $p=$ $0,003 ; 95 \%$ IC $=5,52$ e 34,94$)$ e entre G2 e G4 ( $p=0,001 ; 95 \%$ IC $=12,85$ e 39,94$) ; 3^{\circ}$ ) na dupla tarefa cognitiva: entre $\mathrm{G} 2$ e G1 ( $p=0,013 ; 95 \% \mathrm{IC}=35,83$ e 65,05), entre $\mathrm{G} 2$ e $\mathrm{G} 3$ ( $p=$ $0,016 ; 95 \%$ IC $=4,78$ e 61,78$)$ e entre G2 e G4 ( $p=0,001 ; 95 \%$ IC $=13,10$ e 65,59$)$.

No tocante ao tempo necessário para execução da tarefa, também ficou evidenciada diferença entre grupos nas situações normal $(F=4,136 ; p=0,011)$, de dupla tarefa motora ( $F$ $=5,472 ; p=0,02)$ e de dupla tarefa cognitiva $(F=6,166 ; p=$ 0,001 ). Acerca da análise aos pares, comprovou-se diferença estatisticamente significante nas seguintes situações: $\left.1^{\circ}\right)$ no teste TUG normal: entre G2 e G3 ( $p=0,041 ; 95 \%$ IC $=0,58$ e $37,59)$ e entre $G 2$ e $G 4$ ( $p=0,007 ; 95 \% I C=4,71$ e 38,79); a análise entre $G 2$ e $G 1$ apontou tendência de significância ( $p$ $=0,065 ; 95 \% \mathrm{IC}=-0,82$ e 37,62$) ; 2^{\circ}$ ) na dupla tarefa motora: entre $G 2$ e $G 1$ ( $p=0,036 ; 95 \%$ IC = 0,91 e 36,79), entre $G 2$ e $G 3$ $(p=0,018 ; 95 \% \mathrm{IC}=2,67$ e 37,20$)$ e entre $\mathrm{G} 2$ e $\mathrm{G} 4(\mathrm{p}=0,001$; $95 \% \mathrm{IC}=7,75$ e 39,54$\left.) ; 3^{\circ}\right)$ na dupla tarefa cognitiva: entre $G 2$ e $\mathrm{G} 1(\mathrm{p}=0,022 ; 95 \% \mathrm{IC}=2,33$ e 40,59), entre $\mathrm{G} 2$ e $\mathrm{G} 3$ ( $p=$ $0,021 ; 95 \% \mathrm{IC}=2,45$ e 39,28$)$ e entre $\mathrm{G} 2$ e $\mathrm{G} 4(\mathrm{p}=0,001 ; 95 \%$ IC $=10,15$ e 44,36).

As análises longitudinais dos grupos G1, G2, G3 e G4, envolvendo as situações de teste normal, dupla tarefa motora e dupla tarefa cognitiva, encontram-se na tabela 3.

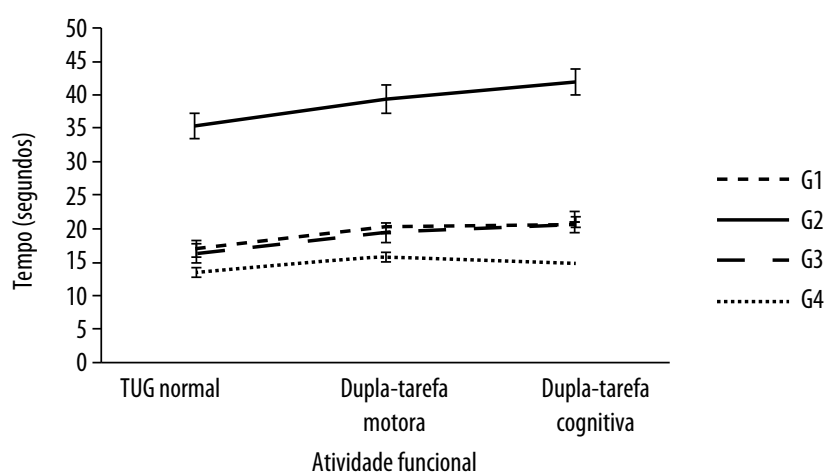

Figura 3. Tempo (segundos) utilizados para a realização da atividade com e sem dupla tarefa. 
Tabela 3. Intervalo de confiança refletindo análises pareadas dos grupos nas situações de teste normal, dupla tarefa motora e dupla tarefa cognitiva

\begin{tabular}{|c|c|c|c|c|c|c|c|c|c|c|}
\hline \multirow{3}{*}{$\begin{array}{l}\text { Variáveis } \\
\text { Passos }\end{array}$} & \multirow{3}{*}{$\begin{array}{c}\text { Grupos } \\
\text { G1 }\end{array}$} & \multicolumn{3}{|c|}{ Normal versus DTM } & \multicolumn{3}{|c|}{ DTM versus DTC } & \multicolumn{3}{|c|}{ Normal versus DTC } \\
\hline & & \multicolumn{2}{|c|}{$95 \%$ IC } & \multirow{2}{*}{$\frac{p}{0,014}$} & \multicolumn{2}{|c|}{$95 \%$ IC } & \multirow{2}{*}{$\frac{\mathbf{p}}{0,227}$} & \multicolumn{2}{|c|}{$95 \% \mathrm{IC}$} & \multirow{2}{*}{$\frac{\mathbf{p}}{0,173}$} \\
\hline & & $-5,56$ & $-0,80$ & & $-1,06$ & 3,97 & & $-4,34$ & 0,89 & \\
\hline & G2 & $-10,17$ & 6,77 & 0,661 & $-41,17$ & 16,77 & 0,366 & $-44,16$ & 16,36 & 0,326 \\
\hline & G3 & $-4,28$ & 0,10 & 0,060 & $-1,53$ & 3,89 & 0,355 & $-4,79$ & 2,97 & 0,614 \\
\hline & G4 & $-7,05$ & $-1,42$ & 0,006 & $-1,57$ & 7,22 & 0,192 & $-4,14$ & 1,32 & 0,290 \\
\hline \multirow[t]{4}{*}{ Tempo } & G1 & $-6,32$ & $-0,76$ & 0,017 & $-2,26$ & 2,08 & 0,093 & $-5,17$ & $-2,09$ & 0,001 \\
\hline & $\mathrm{G} 2$ & $-11,63$ & 3,63 & 0,266 & $-7,90$ & 2,50 & 0,271 & $-15,47$ & 2,07 & 0,118 \\
\hline & G3 & $-6,12$ & 0,30 & 0,071 & $-3,67$ & 1,31 & 0,316 & $-8,36$ & 0,18 & 0,059 \\
\hline & G4 & $-5,67$ & $-1,62$ & 0,002 & $-1,44$ & 5,79 & 0,220 & $-4,08$ & 1,14 & 0,251 \\
\hline
\end{tabular}

Normal: teste Timed Get Up and Go aplicado sem nenhum distrator; DTM: teste Timed Get Up and Go aplicado com dupla tarefa motora; DTC: teste Timed Get Up and Go aplicado com dupla tarefa cognitiva.

\section{DISCUSSÃO}

A evolução tecnológica, associada ao aumento da qualidade de vida dos sujeitos, tem garantido um envelhecimento cada vez mais ativo e longínquo da população mundial. As doenças crônicas classificadas como "idade-dependentes" recebem destaque, por apresentarem prevalência crescente em nosso meio ${ }^{18}$. Nossos resultados apontam que, de forma geral, os participantes com demência do tipo Parkinson apresentam maior comprometimento nas variáveis cognitivas, funcionais e motoras, em relação aos demais idosos. Diferentemente, os sujeitos com DP sem quadro demencial apresentam respostas cognitivas e funcionais semelhantes às dos idosos controles, apesar de a habilidade motora ter sido pior caracterizada na DP. Já os idosos com DA demonstraram interferência da sua condição clínica sobre todas as questões avaliativas, mas em menor grau do que na condição de DP associada a quadro demencial.

As funções cognitivas são definidas como um conjunto de funções cerebrais superiores vinculadas a atividades de atenção, percepção, memória, raciocínio, juízo, tomada de decisão, imaginação, pensamento e linguagem ${ }^{19}$. Em nosso estudo, focamos a análise cognitiva nas funções pré-frontais (executivas), por esta apresentar grande importância na habilidade de iniciar, organizar e sequenciar o comportamento humano. Como pesquisas prévias comprovam interferência da prática de exercício no aparato cognitivo e motor de idosos com DP e DA, optamos por deixar esta amostra homogênea, incluindo apenas sujeitos sedentários e que não realizassem qualquer tipo de oficina de memória, fisioterapia, musicoterapia ou terapia correlata ${ }^{20,21}$.

Na variável cognitiva, observamos respostas similares entre os indivíduos dos grupos G2 e G3 (ambos com comprometimento cognitivo), bem como entre os grupos $\mathrm{G} 1$ e G4 (ambos sem comprometimento cognitivo). Apesar de a modificação patológica mais marcante na DP ser a degene- ração da pars compacta da substância negra mesencefálica, estudos recentes comprovam que o processo apoptótico sofrido nessa doença envolve lesões neuronais adjacentes aos núcleos da base e que afetam, além do tronco encefálico e do lócus cerúleo, regiões cerebrais superiores ${ }^{22-24}$.

Adicionalmente, nos resultados obtidos por meio dos instrumentos MoCA, TFVS e BAF, os pacientes dos grupos G2 e G3 apresentaram respostas similares, pois a disfunção do lobo frontal é comum em ambas as condições. Ao passo que na demência de Parkinson as alterações pré-frontais decorrem de distúrbios dopaminérgicos decorrentes de projeções da substância negra para o corpo estriado, que reduzem a atividade da alça frontoestriatal e da área tegmental ventral para a região pré-frontal do cérebro, na DA a lesão neural pré-frontal decorre de uma hipoperfusão cerebral iniciada no núcleo basal de Meynert, que, por meio de emaranhados neurofibrilares associados ao processamento anormal da proteína precursora beta-amiloide, causa situação de angiopatia amiloidal25,26.

Sobre a análise da variável cognitiva envolvendo os grupos G1 e G4, estudos apontam que mais de 50\% dos pacientes com DP sem demência têm alguma forma de alteração cognitivo-comportamental, como déficits de memória, apatia, disfunções executivas, sintomas depressivos e desempenho cognitivo globalmente prejudicado ${ }^{27,28}$. Apesar do indicativo presente na literatura, é possível afirmar que a similaridade de respostas dos grupos G1 e G4 sobre os instrumentos cognitivos aplicados vislumbra que não há indícios de lesões pré-frontais no G1.

As atividades da vida diária, classificadas como básicas quando envolvem situações de autocuidado (por exemplo, alimentar-se, banhar-se e vestir-se) e como instrumentais quando relacionam tarefas cotidianas mais complexas (por exemplo, realizar compras, atender telefone e utilizar meios de transporte), foram avaliadas e confrontadas ${ }^{29}$. Em relação aos instrumentos aplicados, é importante dizer que o EK 
concentra sua avaliação nas atividades básicas, O IP envolve atividades instrumentais e a EA abrange ambas as atividades: básicas e instrumentais.

Pelos resultados obtidos na avaliação das atividades básicas, constatou-se que o grupo de pacientes com demência de Parkinson apresentou pior rendimento que os demais grupos, demonstrando importante interferência do declínio cognitivo-motor na realização das atividades básicas da vida diária. Os escores dos pacientes com DA e DP sem demência, apesar de indicarem maior dificuldade destes do que dos participantes do grupo controle, não constituíram diferença significante. Esse achado pode ser explicado pelo fato de as atividades básicas exigirem menor ativação das funções cognitivas pré-frontais do que as atividades instrumentais da vida diária - sendo estas últimas utilizadas até mesmo no rastreio de quadros demenciais ${ }^{30}$. Assim sendo, é possível inferir que os instrumentos de avaliação das atividades básicas talvez não tenham sido tão sensíveis quanto às escalas que mensuram as atividades instrumentais.

Especificamente no que tange às atividades instrumentais, o grupo G3 apresentou pior comprometimento em relação aos participantes dos demais grupos. Conquanto esperássemos resultados semelhantes do grupo de pacientes com demência de Parkinson (G2), acreditamos que o maior erro-padrão desse grupo pode ter influenciado nessa variável, traduzindo uma heterocedasticidade dos resultados. Mesmo assim, é fundamental reforçar que os escores do IP foram sensivelmente maiores no G2 e G3, indicando uma associação direta do declínio cognitivo com o declínio funcional ${ }^{31}$.

Sobre a associação de declínio cognitivo e a perda da independência do sujeito na realização das atividades do dia a dia, Rajan et al. ${ }^{32}$ observaram que houve declínio constante na realização das atividades básicas e instrumentais da vida diária dos idosos nos anos subsequentes à avaliação inicial, associado à diminuição de escore cognitivo deles. Esse fato revela importante associação entre ambas as variáveis, mas não deixa claro o proeminente fator causal: se foi o declínio funcional que potencializou o declínio das funções cognitivas ou se foi o déficit cognitivo que acelerou o declínio da funcionalidade em idosos.

Ainda em relação à discussão anterior, tendo em vista os resultados de pior desempenho funcional encontrados nos grupos com comprometimento cognitivo (G2 e G3) e os valores similares de funcionalidade encontrados nos grupos sem comprometimento cognitivo (G1 e G4), é possível inferir que o declínio cognitivo, presente na DA e na demência de Parkinson, exerceu maior interferência nas atividades funcionais da vida diária do que distúrbios motores, como a DP, que apresentou resultados próximos aos dos idosos controles. De qualquer forma, as dificuldades que os pacientes apresentam ao realizar as atividades cotidianas - sejam elas instrumentais ou básicas - são graves, pois podem causar isolamento e exclusão social deles, interferindo na sua qualidade de vida ${ }^{33}$.

A habilidade motora dos sujeitos envolvendo tarefas cotidianas simples promove importante ativação da área motora primária do cérebro (giro pré-central), necessária para realização da tarefa, e pouca ativação das funções cognitivas préfrontais. Já as atividades mais complexas, por envolverem a necessidade de planejamento, sequenciamento e execução do movimento, causam maior ativação das funções executivas $^{34}$. Nosso estudo comprovou que tanto na situação envolvendo atividade motora simples (TUG aplicado sem nenhum distrator) quanto na atividade motora mais complexa (TUG aplicado em situações de dupla tarefa), o grupo de pacientes com comprometimento cognitivo-motor (demência de Parkinson) apresentou pior rendimento na execução das atividades, seguido pelo grupo de pacientes com DA e DP. O grupo controle, formado por idosos saudáveis, pouco sofreu influência do teste quando submetido à situação de dupla tarefa, qualquer que fosse o distrator.

Ao constatar comprometimento funcional maior do grupo de pacientes com demência de Parkinson, seguidos pelos sujeitos com DA e DP, observou-se resposta similar à encontrada por Giovannetti et al. ${ }^{35}$. Para esses autores ${ }^{35}$, pacientes com doenças neurodegenerativas apresentam erros funcionais constantes, classificados como "erros de omissão", em que a resposta motora é tardia, e "erros de comissão", em que a resposta motora é incorretamente executada, vindo a ter grande prejuízo de qualidade de momento e risco aumentado de quedas.

Ainda comparando com outros trabalhos, o presente estudo corrobora os achados clínicos obtidos previamente, ao indicar que pacientes com quadro de demência na DP apresentam maior prejuízo do desempenho motor quando comparados a pacientes com DP sem demência ${ }^{36}$. As alterações motoras encontradas nesses pacientes justificam o comprometimento na postura e equilíbrio e interferem diretamente nas atividades funcionais que são realizadas durante a bipedestação ${ }^{37}$.

Por fim, cabe destacar que, na medida em que as doenças geradoras de limitações físicas afetam a qualidade de vida dos indivíduos, ao tornarem dificultosa a realização das atividades instrumentais da vida diária, as afecções cognitivas podem não apresentar tal efeito, pois o declínio cognitivo do paciente tende a afetar a sua noção de qualidade de vida ${ }^{38}$.

$\mathrm{Na}$ interpretação dos resultados encontrados, deve ser levado em consideração que os participantes encontravam-se em graus moderados em ambas as enfermidades. Além disso, não incluímos casos de DA ou DP precoce, por entendermos que tais casos poderiam representar outliers perante os demais participantes.

Conquanto possa se arguir sobre o pequeno tamanho amostral, deve-se considerar a dificuldade de recrutamento dos sujeitos, bem como o fato de ambas as patologias 
apresentarem diagnóstico complexo, exigindo a integração da avaliação clínica com exames laboratoriais, nem sempre disponíveis. Na DA, por exemplo, o diagnóstico definitivo é realizado por meio da autópsia cerebral. Em vida, a utilização de exames de neuroimagem estrutural (volumetria do hipocampo, por exemplo) e de neuroimagem funcional (espectroscopia por ressonância magnética) auxilia no diagnóstico mais próximo do correto. Já na DP, a dificuldade do diagnóstico está em diferenciar DP idiopática das síndromes parkinsonianas secundárias ao comprometimento cerebral.

Ainda que alguns pesquisadores possam questionar possível interferência da baixa escolaridade dos sujeitos sobre os resultados, deve-se considerar que utilizamos testes cognitivos e motores amplamente utilizados na literatura e que envolvem tarefas simples e de fácil compreensão.

Apesar do alto índice de sujeitos não incluídos (considerando o total de pacientes inicialmente selecionados), optamos por tornar a amostra o mais homogênea possível, a fim de evitar a inclusão de vieses de seleção e controlar os erros tipos 1 e 2 .

\section{CONCLUSÃO}

Este estudo observou que os distúrbios pré-frontais, presentes na demência de Parkinson e na DA, repercutem negativamente nas atividades funcionais da vida diária e nas habilidades psicomotoras dos sujeitos. Quando não vinculados a quadro demencial, os pacientes com DP apresentaram escores cognitivos pré-frontais e independência funcional semelhantes aos de idosos saudáveis.

A habilidade motora em situações de tarefas duplas encontra-se prejudicada nos pacientes com DP (com e sem quadro demencial) e DA. Por remeter à necessidade de ativação de funções executivas, pacientes com déficits cognitivos apresentam maior dificuldade na realização de tarefas duplas, em comparação com pacientes com déficits eminentemente motores. A realização de novos estudos que abordem a interferência das funções pré-frontais nas doenças neurodegenerativas é importante, sobretudo quando associados a técnicas de neuroimagem funcional.

\section{AGRADECIMENTOS}

Agradecemos à Coordenação de Aperfeiçoamento de Pessoal de Nível Superior (Capes), pelo apoio e suporte financeiro, e ao grupo de pesquisa "Avaliação e Intervenção em Fisioterapia Neurofuncional" da Universidade Federal de Mato Grosso do Sul (UFMS), Conselho Nacional de Desenvolvimento Científico e Tecnológico (CNPq).

\section{CONFLITOS DE INTERESSE}

Não há.

\section{CONTRIBUIÇÕES INDIVIDUAIS}

Lilian Assunção Felippe e Gustavo Christofoletti - Participaram da concepção e do desenho do estudo.

Lilian Assunção Felippe, Renata Terra de Oliveira e Suhaila Mahmoud Smaili Santos - Realizaram as pesquisas bibliográficas.

Lilian Assunção Felippe, Renata Terra de Oliveira $\mathrm{e}$ Milena Garcia-Participaram da coleta dos dados.

Gustavo Christofoletti e Tânia Cristina Dias da Silva

-Hamu - Procederam à análise estatística.

Lilian Assunção Felippe, Suhaila Mahmoud Smaili Santos, Tânia Cristina Dias da Silva-Hamu e Gustavo Christofoletti - Escreveram o primeiro rascunho do manuscrito.

\section{REFERÊNCIAS}

1. Hung WW, Ross JS, Boockyar KS, Siu AL. Association of chronic diseases and impairments with disability in older adults: a decade of change? Med Care. 2012;50(6):501-7.

2. Torrão AS, Café-Mendes $C C$, Real CC, Hernandes MS, Ferreira AFB, Santos TO, et al. Different approaches, one target: understanding cellular mechanisms of Parkinson's and Alzheimer's diseases. Rev Bras Psiquiatr. 2012;34:S194-205.

3. Christofoletti G, Freitas RT, Cândido ER, Cardoso CS. Eficácia de tratamento fisioterapêutico no equilíbrio estático e dinâmico de pacientes com doença de Parkinson. Fisioter Pesqui. 2010;17(3):259-63.

4. Martyr A, Clare L. Executive function and activities of daily living in Alzheimer's disease: a correlational meta-analysis. Dement Geriatr Cogn Disord. 2012;33(2-3):189-203.

5. Montero-Odasso M, Verghese J, Beauchet 0l, Hausdorff J. Gait and cognition: a complementary approach to understanding brain function and the risk of falling. J Am Geriatr Soc. 2012;60(11):2127-36.

6. Hoehn MM, Yahr MD. Parkinsonism: onset, progression and mortality. Neurology. 1967;17(5):427-42

7. Fahn S, Elton RL. Unified Parkinson's Disease Rating Scale. In: Fahn S, Marsden CD, Goldstein M, Calne DB (eds.). Recent developments in Parkinson's disease. New York: MacMilIan; 1987. p. 153-63.

8. Hughes CP, Berg L, Danziger WL, Coben LA, Martin RL. A new clinical scale for the staging of dementia. Br J Psychiatr. 1982;140:566-72.

9. Folstein MF, Folstein SE, McHugh PR. "Mini-mental state". A practical method for grading the cognitive state of patients for the clinician. J Psychiatr Res. 1975;12(3):189-98.

10. Nasreddine Z, Phillips NA, Bédirian V, Charbonneau S, Whitehead V, Collin I, et al. The Montreal Cognitive Assessment, MoCA: a brief screening tool for mild cognitive impairment. J Am Geriatr Soc. 2005;53:695-9.

11. Lezak MD. Neuropsychological assessment. 3rd ed. New York: Oxford University Press; 1995.

12. Dubois B, Slachevsky A, Litvan I, Pillon B. The FAB: a frontal assessment battery at bedside. Neurology. 2000;55:1621-6.

13. Podsiadlo D, Richardson S. The "Timed Up and Go": a test of basic functional mobility for frail elderly persons. J Am Geriatr Soc. 1991;39(2):142-8. 
14. Ries JD, Ecternch JL, Nof L, Gagnon-Blodgett M. Test-retest reliability and minimal detectable change scores for the timed "up and go" test, the six minute walk test, and gait speed in people with Alzheimer's disease. Phys Ther. 2009;89(6):569-79.

15. Andreotti RA, Okuma SS. Validação de uma bateria de testes de atividades da vida diária para idosos fisicamente independentes. Rev Paul Educ Fís. 1999;3:46-66.

16. Katz S, Stroud MW 3rd. Functional assessment in geriatrics: a review of progress and directions. J Am Geriatr Soc. 1989;37(3):267-71.

17. Pfeffer Rl, Kurosaki TT, Harrh CH, Chance JM, Filos S. Measurement of functional activities in older adults in the community. J Gerontol. 1982;37:323-9.

18. Chan FW, Wong FY, So WY, Kung K, Wong CK. How much do elders with chronic conditions know about their medications? BMC Geriatr. 2013;13:50

19. De La Fuente A, Xias S, Branch C, Li X. A review of attention-deficit/hyperactivity from the perspective of brain networks. Front Hum Neurosci. 2013;7:192.

20. Andrade LP, Gobbi LTB, Coelho FGM, Christofoletti G, Riani-Costa JL, Stella F. Benefits of multimodal exercise intervention for postural control and frontal cognitive functions in individuals with Alzheimer's disease: a controlled trial. J Am Geriatr Soc. 2013;61(11):1919-26.

21. Christofoletti G, Oliani MM, Bucken-Gobbi LT, Gobbi S, Beinotti F, Stella F. Physical activity attenuates neuropsychiatric disturbances and caregiver burden in patients with dementia. Clinics. 2011;66(4):613-8.

22. Biundo R, Calabrese M, Weis L, Facchini S, Ricchieri G, Gallo P, et al. Anatomical correlates of cognitive functions in early Parkinson's disease patients. PLoS One. 2013;8(5):e64222.

23. Hildebrand H, Fink F, Kastrup A, Haupts M, Eling P. Cognitive profiles of patients with mild cognitive impairment or dementia in Alzheimer's or Parkinson's disease. Dement Geriatr Cogn Dis Extra. 2013;3(1):102-12.

24. Carbon M, Marie RM. Functional imaging of cognition in Parkinson's disease. Curr Opin Neurol. 2003;16:475-80

25. Morbelli S, Perneczky R, Drzezga A, Frisoni GB, Caroli A, van Berckel BN, et al. Metabolic networks underlying cognitive reserve in prodromal Alzheimer disease: a European Alzheimer Disease Consortium Project. J Nucl Med. 2013;54(6):894-902.
26. Pillon B, Czernecki V, Dubois B. Dopamine and cognitive function. Curr Opin Neurol. 2003;16(Suppl 2):S17-22

27. Janvin C, Aarsland D, Larsen JP, Hugdahl K. Neuropsychological profile of patients with Parkinson's disease without dementia. Dement Geriatr Cogn Disord. 2003;15:126-31.

28. Christofoletti G, Cândido ER, Olmedo L, Miziara SRB, Beinotti F. Efeito de uma intervenção cognitivo-motora sobre os sintomas depressivos de pacientes com doença de Parkinson. J Bras Psiquiatr. 2012;61(2):78-83

29. Jacobs JM, Maaravi Y, Cohen A, Bursztyn M, Ein-Mor E, Stessman J. Changing profile of health and function from age 70 to 85 . Gerontology. 2012;58(4):313-21.

30. Marshall GA, Amariglio RE, Sperling RA, Rentz DM. Activities of daily living: where do they fit in the diagnosis of Alzheimer's disease? Neurodegener Dis Manag. 2012;2(5):483-91.

31. Brito TRP, Costa RS, Pavarini SFI. Idosos com alteração cognitiva em contexto de pobreza: estudando a rede de apoio social. Rev Esc Enferm USP. 2012;46(4):906-13.

32. Rajan KB, Hebert LE, Scherr PA, Mendes de Leon CF, Evans DA. Disability in basic and instrumental activities of daily living in associated with faster rate of decline in cognitive function o folder adults. J Gerontol A Biol Sci Med Sci. 2013;68(5):624-30.

33. Gure TR, Langa KM, Fisher GG, Piette JD, Plassman BL. Functional limitations in older adults who have cognitive impairment without dementia. J Geriatr Pshyciatry Neurol. 2013;26(2):78-85.

34. Ohsugi H, Ohgi S, Shigemori K, Schneider EB. Differences in dual-task performance and prefrontal cortex activation between younger and older adults. BMC Neurosci. 2013;14:10.

35. Giovannetti T, Britnell P, Brennan L, Siderowf L, Grossman M, David J, et al. Everyday action impairment in Parkinson's disease dementia. J Int Neuropsychol Soc. 2012;18(5):787-98.

36. Agostino R, Jones JN, Hallett M. Motor skill learning in Parkinson's disease. J Neurol Sci. 1996;139:218-26

37. Morris ME. Movement disorders in people with Parkinson disease: a model for physical therapy. Phys Ther. 2000;80:578-97.

38. Weiss EM, Papousek I, Fink A, Matt T, Marksteiner J, Deisenhammer EA. Quality of life in mild cognitive impairment, patients with different stages of Alzheimer disease and healthy control subjects. Neuropsychiatr. 2012;26(2):72-7. 